\title{
Investigation into in vitro anti-leishmanial combinations of calcium channel blockers and current anti-leishmanial drugs
}

\author{
Juliana Quero Reimão, André Gustavo Tempone/ ${ }^{+}$ \\ Departamento de Parasitologia, Instituto Adolfo Lutz, Av. Dr. Arnaldo 351, 01246-000 São Paulo, SP, Brasil
}

\begin{abstract}
The need for drug combinations to treat visceral leishmaniasis (VL) arose because of resistance to antimonials, the toxicity of current treatments and the length of the course of therapy. Calcium channel blockers (CCBs) have shown anti-leishmanial activity; therefore their use in combination with standard drugs could provide new alternatives for the treatment of VL. In this work, in vitro isobolograms of Leishmania (Leishmania) chagasi using promastigotes or intracellular amastigotes were utilised to identify the interactions between five CCBs and the standard drugs pentamidine, amphotericin B and glucantime. The drug interactions were assessed with a fixed ratio isobologram method and the fractional inhibitory concentrations (FICS), sum of FICs ( $F F I C s)$ and the overall mean SFIC were calculated for each combination. Graphical isobologram analysis showed that the combination of nimodipine and glucantime was the most promising in amastigotes with an overall mean $\Sigma F I C$ value of 0.79. Interactions between CCBs and the anti-leishmanial drugs were classified as indifferent according to the overall mean $\mathrm{FIC}$ and the isobologram graphic analysis.
\end{abstract}

Key words: leishmaniasis - Leishmania therapy - calcium channel blockers - drug combinations - isobologram

Visceral leishmaniasis (VL) is a disseminated protozoan infection caused by the Leishmania (Leishmania) donovani complex (Chappuis et al. 2007). The zoonotic form, in which dogs are the main reservoir in urban areas, is widely distributed in the Mediterranean basin, China, the Middle East and South America and is caused by Leishmania (Leishmania) infantum/Leishmania (Leishmania) chagasi (Herwaldt 1999, Chappuis et al. 2007). Although the disease is endemic in more than 60 countries with 200 million people at risk, $90 \%$ of the 500,000 cases are found in five countries: India, Bangladesh, Nepal, Sudan and Brazil (van Griensven et al. 2010).

In the past 70 years, the therapeutic arsenal for the treatment of VL has been extremely limited, consisting of the pentavalent antimonials (sodium stibogluconate and meglumine antimoniate), amphotericin B (and its lipid formulations) and miltefosine (Alvar et al. 2006). These drugs have crucial disadvantages, such as the length of treatment required, painful injection, toxicity, the emergence of resistance, dose-limiting nephrotoxicity, heat instability, high cost and poor patient compliance (van Griensven et al. 2010).

Calcium channel blockers (CCBs) are drugs used to treat heart diseases. This class of drugs inhibits the action of the calcium channels in cell membranes and potential indications are not limited to cardiovascular diseases. The class consists of three chemically distinct

Financial support: FAPES (08/09260-7), CNPq (to AGT) and FAPESP (08/11434-3 to JQR)

+ Corresponding author: atempone@ial.sp.gov.br

Received 4 July 2011

Accepted 25 October 2011 structural families: phenylalkylamines (e.g., verapamil), dihydropyridines (e.g., amlodipine, nimodipine) and benzothiazepines (e.g., diltiazem) (Motro et al. 2001). Considering that drug repurposing, also referred to as drug repositioning, is a promising approach to discover novel drug candidates to treat neglected diseases, the anti-parasitic activity of CCBs has been studied. It was demonstrated that nifedipine blocks the binding of Leishmania amastigotes to macrophages previously treated with the drug, suggesting that calcium has a role during the infection of macrophages by new parasites (Misra et al. 1991). There are also reports of the in vitro and in vivo activity of amlodipine and lacidipine against L. (L.) donovani (Palit \& Ali 2008).

Based on their cardiac and peripheral activity, CCBs have been divided into the following classes (Singh 1986): (i) type I agents, exemplified by verapamil and related drugs (e.g., tiapamil, galopamil) and diltiazem (antiarrhythmics), (ii) type II agents, including nifedipine and other dihydropyridines, which are potent peripheral vasodilators with selective action on sympathetic reflexes, (iii) type III agents, including flunarizine and cinnarizine (piperazine derivatives), which are potent dilators of peripheral vessels, and (iv) type IV agents show broad pharmacological action (e.g., perhexiline, lidoflazine and bepridil), which includes blocking the calcium flow in the heart and peripheral vessels and other electrophysiological actions.

The goals of the drug combinations are to prevent or delay the onset of resistance and relapses and also to increase the efficiency or reduce the course of treatment. These combinations have been the standard therapy of various viral, bacterial and parasitic diseases (White 1999, Olliaro \& Taylor 2003). The need to investigate drug combinations for the therapy of leishmaniasis has been noted by several authors (Bryceson 2001, Sundar 2001, van Griensven et al. 2010). Drug combinations 
have been highlighted for VL in response to the need to overcome antimonial resistance, prolong the therapeutic lifespan of the drugs, shorten the length of treatment, improve patient compliance and increase costeffectiveness (Olliaro 2010).

Based on previous reports that demonstrate the antileishmanial activity of several CCBs (Tempone et al. 2009, Reimão et al. 2010), their in vitro activity when used in combination with standard drugs was explored with the goal of finding new alternatives for the treatment of leishmaniasis.

\section{MATERIALS AND METHODS}

Material - Sodium dodecyl sulphate, 3-(4,5-dimethylthiazol-2-yl)-2,5-diphenyltetrazolium bromide (MTT) (Thiazol blue), M-199 and Roswell Park Memorial Institute (RPMI)-PR-1640 medium (without phenol red) were purchased from Sigma (St. Louis, MO, USA). Pentavalent antimony (glucantime, Aventis-Pharma-Brazil) and pentamidine (Sideron, Brazil) were used as standard drugs. Other analytical reagents were purchased from Sigma unless stated otherwise.

Animals - Golden hamsters (Mesocricetus auratus) and $\mathrm{BALB} / \mathrm{c}$ mice were obtained from the Adolfo Lutz Institute of São Paulo and kept in sterile boxes with absorbent material while receiving food and water ad libitum. Golden hamsters were infected each month with amastigotes from the spleen to maintain the strain. BALB/c mice were used for obtaining peritoneal macrophages.

Parasites and macrophages - L. (L.) chagasi (MHOM/ $\mathrm{BR} / 1972 / \mathrm{LD}$ ) promastigotes were grown in M-199 medium supplemented with $10 \%$ foetal calf serum (FCS) and $0.25 \%$ haemin at $24^{\circ} \mathrm{C}$ without addition of antibiotics. $L$. (L.) chagasi amastigotes were obtained by differential centrifugation from spleens of previously infected golden hamsters. The number of parasites was determined (Stauber 1958) 60-70 days after infection. Macrophages were collected from the peritoneal cavity of BALB/c mice by washing with RPMI-1640 medium supplemented with $10 \%$ FCS and were maintained in a $5 \% \mathrm{CO}_{2}$-humidified incubator at $37^{\circ} \mathrm{C}$ (Tempone et al. 2008).

Determination of drug interactions - The interactions between drugs were evaluated in vitro by a modified isobologram method (Fivelman et al. 2004). The predetermined $50 \%$ inhibitory concentration $\left(\mathrm{IC}_{50}\right)$ values were used to determine the maximum concentrations of individual drugs, assuring that the $\mathrm{IC}_{50}$ was in the fourth point of the serial dilution. Drugs were dissolved in dimethyl sulphoxide (DMSO) and diluted with medium. The highest concentrations of the solutions were prepared in proportions of 5:0, 4:1, 3:2, 2:3, 1:4 and 0:5 of CCB and standard drug, respectively, which were serially diluted (base 2) to the seventh well of the microplate in duplicate. Two separate experiments were performed for each drug combination and susceptibility assay.

Promastigotes assay - Promastigotes were counted in a Neubauer haemocytometer and seeded at $1 \times 10^{6} /$ well with a final volume of $200 \mu \mathrm{L}$. The initial concentrations used were $12 \mu \mathrm{g} / \mathrm{mL}$ for amlodipine, $12 \mu \mathrm{g} / \mathrm{mL}$ for bepridil, $100 \mu \mathrm{g} / \mathrm{mL}$ for lercanidipine, $100 \mu \mathrm{g} / \mathrm{mL}$ for nicardipine, $280 \mu \mathrm{g} / \mathrm{mL}$ for nimodipine, $1.5 \mu \mathrm{g} / \mathrm{mL}$ for pentamidine and $0.4 \mu \mathrm{g} / \mathrm{mL}$ for amphotericin B. Controls with DMSO and without drugs were performed. The plates were incubated for $24 \mathrm{~h}$ at $24^{\circ} \mathrm{C}$ and the viability of promastigotes was verified by the MTT assay (Tada 1986). The data analysis was performed with Graph Pad Prism 5.0 software. One hundred per cent viability was expressed based on the optical density of control promastigotes after normalisation.

Intracellular amastigotes assay - Macrophages were collected from the peritoneal cavity of BALB/c as described above and added to 16 well plates $\left(\mathrm{NUNC}^{\circledR}\right)$ at $5 \mathrm{x}$ $10^{4} /$ well. Plates were incubated at $5 \% \mathrm{CO}$ for $24 \mathrm{~h}$ at $37^{\circ} \mathrm{C}$. L. (L.) chagasi amastigotes extracted from the spleens and separated by differential centrifugation were added to macrophages at a ratio of 10:1 (amastigotes:macrophage). After $24 \mathrm{~h}$, extracellular parasites were removed by washing and fresh medium containing the different fixed-ratio solutions was added and incubated at $37^{\circ} \mathrm{C}$ for a period of $120 \mathrm{~h}$. Further medium changes with fresh drug were carried out after $72 \mathrm{~h}$. The initial concentrations used were $21 \mu \mathrm{g} / \mathrm{mL}$ for amlodipine, $72 \mu \mathrm{g} / \mathrm{mL}$ for nimodipine, $240 \mu \mathrm{g} / \mathrm{mL}$ for glucantime and $0.2 \mu \mathrm{g} / \mathrm{mL}$ for amphotericin B. At the end of the assay, the slides were stained with Giemsa and observed under an optical microscope. The $\mathrm{IC}_{50}$ was determined by counting 200 macrophages per well and assessing the number of infected macrophages (Yardley \& Croft 2000).

Determination of fractional inhibitory concentrations (FICs) index, isobologram construction and classification of the nature of interaction - FICs and the sum of FICs ( $\Sigma$ FICs) were calculated as follows: FIC of drug $\mathrm{A}=\mathrm{IC}_{50}$ of drug $\mathrm{A}$ in combination/ $/ \mathrm{IC}_{50}$ of drug A alone. The same equation was applied to the partner drug (drug B). $\Sigma$ FICs $=$ FIC drug A + FIC drug B. An overall mean $\Sigma F I C$ was calculated for each combination and used to classify the nature of interaction as follows: synergy defined the mean $\Sigma$ FIC $\leq 0.5$, indifference the mean $\Sigma$ FIC between $>0.5$ and $\leq 4$ and antagonism the mean $\Sigma F I C>$ 4 (Odds 2003). Isobolograms were constructed plotting the standard error of the mean (SEM) for each component of the dosage combination (Gessner 1995).

Statistical analysis - The data obtained represented the mean and standard deviation of duplicate samples from two independent assays. The $\mathrm{IC}_{50}$ values were calculated using sigmoid dose-response curves with Graph Pad Prism 5.0 software and the 95\% confidence intervals were included.

Ethics - All procedures performed on animals were approved by the Ethical Committee on Research of the Adolfo Lutz Institute/Pasteur Institute and were in agreement with the Guidelines for the Care and Use of Laboratory Animals from the National Academy of Sciences.

\section{RESULTS}

Evaluation of the anti-leishmanial activity of single drugs - L. (L.) chagasi was susceptible to all tested CCBs with $\mathrm{IC}_{50}$ values that ranged from $0.8-30 \mu \mathrm{g} / \mathrm{mL}$. As dem- 
onstrated by the mitochondrial oxidative metabolism of parasites (MTT method), the tested drugs showed a leishmanicidal effect by killing $100 \%$ of parasites at the highest tested concentrations. These $\mathrm{IC}_{50}$ values were obtained for the single drugs against promastigotes and are shown in Table I. Amlodipine was the most active CCB against both promastigotes and amastigotes.

Combination studies - The combination of amlodipine, bepridil, lercanidipine, nicardipine and nimodipine with the anti-leishmanial drugs amphotericin B and pentamidine in $L$. (L.) chagasi promastigotes indicated an indifferent interaction. This was demonstrated by analysis of the overall mean $\Sigma$ FICs, which ranged from 1.13-1.7 (Table II). According to the graphic analysis of the isobolograms using promastigotes (Fig. 1), indifference was observed within all combinations because all points $\left(\mathrm{P}_{2}-\mathrm{P}_{4}\right.$, corresponding to the proportions of $4: 1,3: 2$, $2: 3$ and 1:4 of CCB and standard anti-leishmanial drug, respectively) are located above the additivity line. The combination of amlodipine and nimodipine with glucantime and amphotericin B in $L$. (L.) chagasi intracellular amastigotes was classified as indifferent according the overall mean $\Sigma$ FICs, which ranged from 0.79-1.43 (Table II). The graphic analysis of the isobologram (Fig. 2) showed that, in spite of some points being located below the additivity line (point $\mathrm{P}_{2}$ of amlodipine:amphotericin $\mathrm{B}$; point $\mathrm{P}_{5}$ of amlodipine:glucantime and points $\mathrm{P}_{3}$ and $\mathrm{P}_{5}$ of nimodipine:glucantime), the SEM should also be considered in the analysis. The combination of nimodipine and glucantime (Fig. 2D) showed synergy in three of the points in the isobologram, with the most synergic effect seen in the point $\mathrm{P}_{4}$ (ratio 2:3 of nimodipine:glucantime). However, according to the adopted classification, this drug combination was not considered synergic because the overall mean $\Sigma F I C$ of this combination was 0.79 (Table II).

\section{DISCUSSION}

Infectious diseases such as tuberculosis, leprosy, malaria and acquired immune deficiency syndrome were only considered to be under therapeutic control after the introduction of drug combinations. Combinatorial treatments can not only boost the action of the different therapeutic compounds, but they may also help to avoid the development of parasitic resistance (Coura 2009). This is the first report that investigates the in vitro activity of combinations of calcium antagonists and standard drugs for leishmaniasis. Despite the previously observed antileishmanial activity of the CCBs used in the study (Reimão et al. 2010), the overall mean $\Sigma$ FIC and the isobolograms obtained show no physicochemical or biological interactions between the CCBs and the standard drugs.

The isobologram is a graphical representation of the effective dose, or FIC value, of two drugs when administered together. In this graph, the intercepts are the points that define the line of additivity (junction of points $\mathrm{P}_{1}$ and $\mathrm{P}_{6}$ ) (Figs 1,2) and all points in this line are the coordinates that theoretically represent the doses of drug pairs $\left(\mathrm{P}_{2}-\mathrm{P}_{4}\right)$ (Figs 1, 2). This graphical representation provides an overview of the isobologram of theoretical additive doses, but $\Sigma$ FIC values are preferred for statistical analysis (Tallarida et al. 1997).
TABLE I

Activity of individual drugs against Leishmania (Leishmania) chagasi promastigotes and intracellular amastigotes

\begin{tabular}{lcc}
\hline Drug & $\begin{array}{c}\mathrm{IC}_{50} \mu \mathrm{g} / \mathrm{mL} \\
(95 \% \mathrm{CI})\end{array}$ & $\begin{array}{c}\text { L. (L.) chagasi } \\
\text { form }\end{array}$ \\
\hline Amlodipine & $0.85(0.74-0.97)$ & Promastigotes \\
Bepridil & $1.73(1.59-1.88)$ & \\
Lercanidipine & $7.80(6.704-9.075)$ & \\
Nicardipine & $10.59(9.82-11.43)$ & \\
Nimodipine & $30.30(28.32-32.42)$ & \\
Amphotericin B & $0.04(0.03-0.05)$ & \\
Pentamidine & $0.22(0.19-0.26)$ & \\
\hline Amlodipine & $2.93(2.08-4.12)$ & Amastigotes \\
Nimodipine & $26.53(24.87-28.30)$ & \\
Amphotericin B & $0.14(0.13-0.16)$ & \\
Glucantime & $7.25(6.13-8.57)$ & \\
\hline
\end{tabular}

95\% CI: 95\% confidence interval; $\mathrm{IC}_{50}: 50 \%$ inhibitory concentration $(\mu \mathrm{g} / \mathrm{mL})$.

\section{TABLE II}

Overall mean sum of fractional inhibitory concentrations

( $\Sigma$ FIC) of calcium channel blockers and partner drugs against Leishmania (Leishmania) chagasi promastigotes and amastigotes

\begin{tabular}{lcc}
\hline Combination & $\begin{array}{c}\text { Overall } \\
\text { mean } \\
\text { IFIC }\end{array}$ & $\begin{array}{c}\text { L. (L.) } \\
\text { chagasi } \\
\text { form }\end{array}$ \\
\hline Amlodipine + pentamidine & 1.47 & Promastigotes \\
Amlodipine + amphotericin B & 1.40 & \\
Bepridil + pentamidine & 1.19 & \\
Bepridil + amphotericin B & 1.15 & \\
Lercanidipine + pentamidine & 1.49 & \\
Lercanidipine + amphotericin B & 1.70 & \\
Nicardipine + pentamidine & 1.26 & \\
Nicardipine + amphotericin B & 1.32 & \\
Nimodipine + pentamidine & 1.27 & \\
Nimodipine + amphotericin B & 1.13 & \\
\hline Amlodipine + glucantime & 1.19 & Amastigotes \\
Amlodipine + amphotericin B & 1.43 & \\
Nimodipine + glucantime & 0.79 & \\
Nimodipine + amphotericin B & 1.28 & \\
\hline
\end{tabular}

The terminology used to interpret the results of the isobologram is often a subject of debate and confusion (Odds 2003). The terms used to describe results that are neither synergistic nor antagonistic are somewhat complicated. Unfortunately, the term "additive" often holds an inaccurate meaning and can be interpreted as referring to a positive interaction (Odds 2003). For this reason, the terms "synergistic", "indifferent" and "antagonistic" were used for the interpretation of interactions 
A

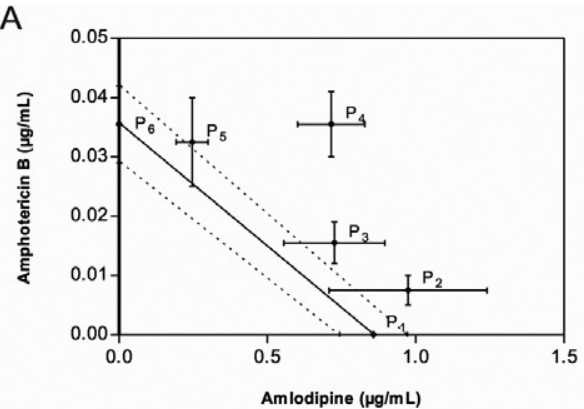

C

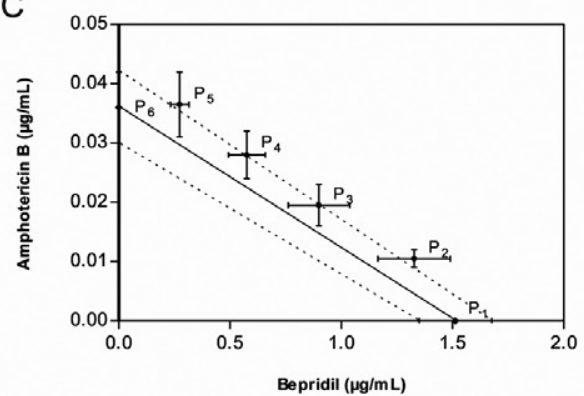

E

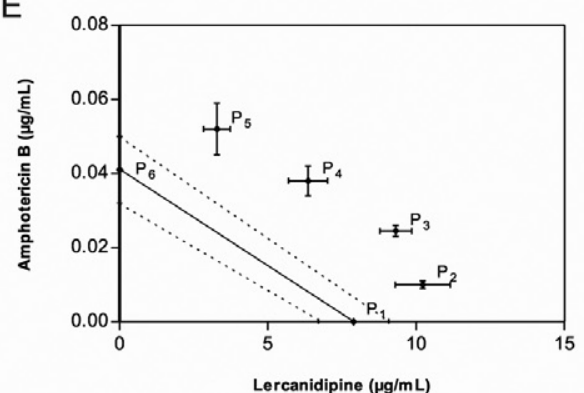

G

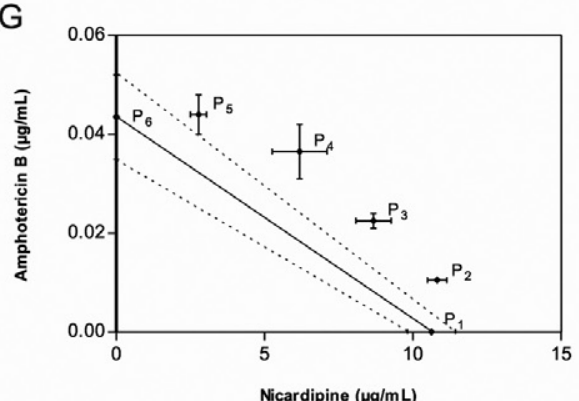

I

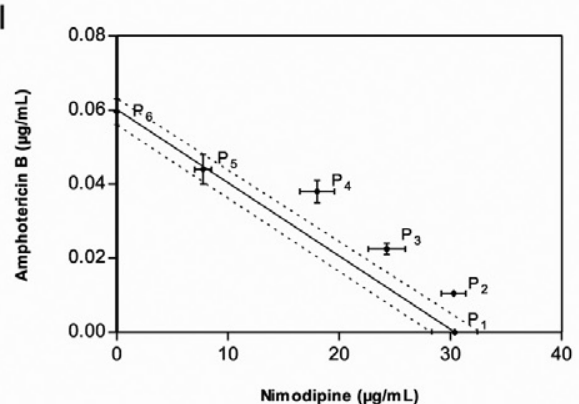

B

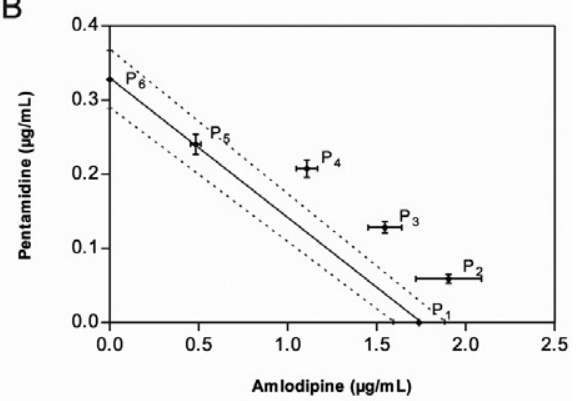

D

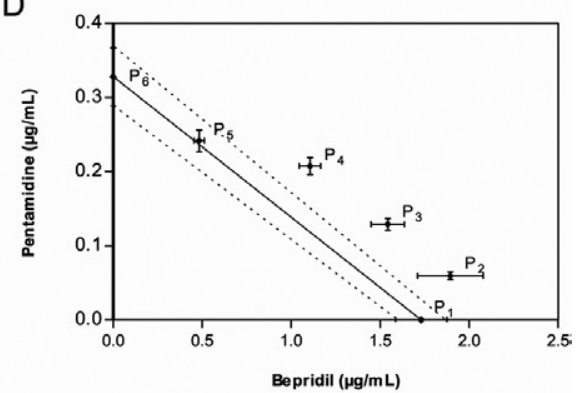

F

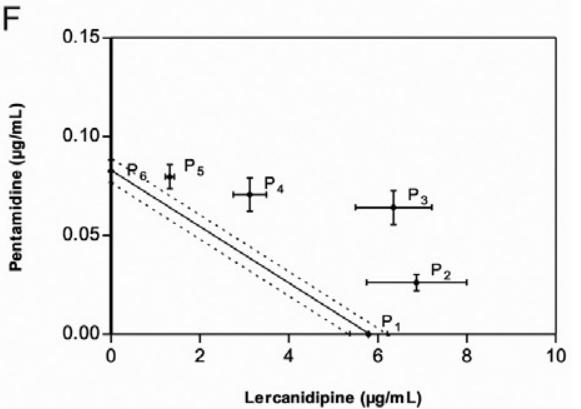

$\mathrm{H}$
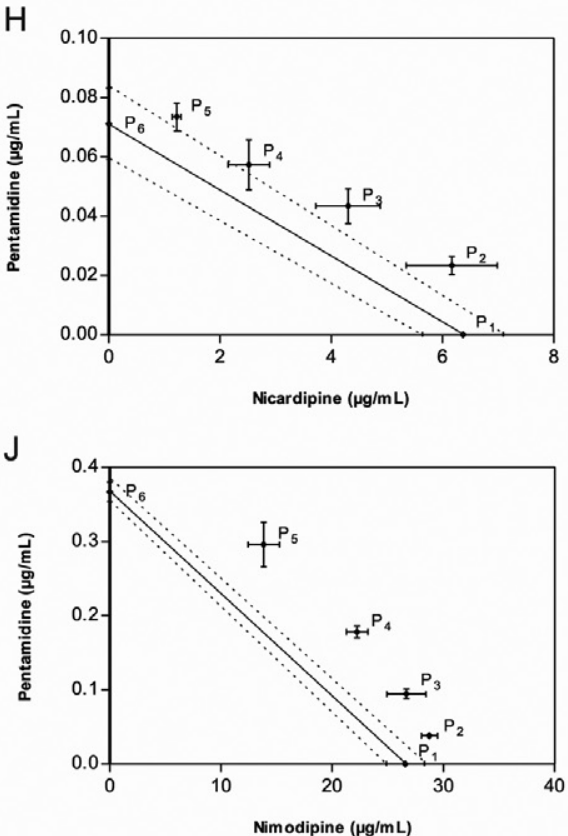

Fig. 1: representative isobolograms of in vitro interactions of calcium channel blockers (CCBs) and the partner drug against Leishmania (Leishmania) chagasi promastigotes. The half maximal inhibitory concentration $\left(\mathrm{IC}_{50}\right)$ of amlodipine, bepridil, lercanidipine, nicardipine and nimodipine was plotted in the abscissa and the $\mathrm{IC}_{50}$ of amphotericin $\mathrm{B}$ and pentamidine was plotted in the ordinate. The plotted points are $\mathrm{IC}_{50} \mathrm{~s}$ of each fixed ratio combination serially diluted. Bars around points correspond to calculated standard errors of the mean. Points $\mathrm{P}_{1}-\mathrm{P}_{6}$ correspond to the proportion of 5:0, 4:1, 3:2, 2:3, 1:4 and 0:5 of CCB and standard anti-leishmanial drug, respectively. The bold line corresponds to the predicted positions of the experimental points for a simple additive effect. The dotted line corresponds to the additivity line range of confidence. 
in this report. The isobologram graph made from FIC values has been widely used to represent the interaction between two drugs (Fivelman et al. 2004). However, it is also possible to use the $\mathrm{IC}_{50}$ values and their respective confidence intervals (Gessner 1995). Thus, the graph with standard error bars can be more informative and was used in the present work.

The FIC values demonstrate that paired combinations of agents can exert inhibitory effects higher than the sum of their effects alone (synergy; FIC < 1.0) or smaller than the sum of their effects alone (antagonism; FIC > 1.0) (Berembaum 1978). However, Odds (2003) proposed the interpretation of FIC data as "synergy" when FIC $\leq 0.5$, "antagonism" when FIC $\geq 4.0$ and "no interaction" when FIC $>0.5<4.0$.

Most relevant information for drug combinations is obtained with the intracellular amastigote assay. However, the use of axenic promastigotes can also provide useful data. It is important to consider that the presence of the host cell (macrophages) could interfere within the synergism/antagonism of the drug combinations during studies against Leishmania. Thus, susceptibility studies using the two parasite stages (promastigotes and intracellular amastigotes) can provide additional information about the distinct action of the drug combinations in addition to that obtained from ordinary macrophage activation.

Calcium antagonists have shown anti-parasitic properties (Misra et al. 1991, Núñez-Vergara et al. 1998, Tempone et al. 2009, Reimão et al. 2010, 2011). Other studies have reported that amlodipine reverses the in vitro chloroquine resistance in Plasmodium falciparum (Basco \& Le Bras
1991) and can also increase chloroquine accumulation inside the infected erythrocytes. Therefore, combination therapy of amlodipine and chloroquine might be a useful therapeutic strategy against chloroquine-resistant malaria (Deloron et al. 1991). In addition, the 1,4-dihydropyridine nicardipine showed in vitro activity against $P$. falciparum $\left(\mathrm{IC}_{50}=4.3 \mu \mathrm{M}\right)$ (Tanabe et al. 1989).

Bepridil, an anti-anginal pyrrolidine drug, demonstrated effectiveness in vitro against a panel of visceral and cutaneous species of Leishmania, with $\mathrm{IC}_{50}$ values ranging from 3-7 $\mu \mathrm{M}$ (Tempone et al. 2009, Reimão et al. 2011). It has also demonstrated activity against other protozoans, such as Toxoplasma gondii (Song et al. 2004), $P$. falciparum $\left(\mathrm{IC}_{50}=2.63 \mu \mathrm{M}\right)$ (Mahmoudi et al. 2006), Trypanosoma cruzi (Reimão et al. 2011) and Entamoeba species (Makioka et al. 2001). However, it lacks activity in an experimental L. (L.) chagasi model (Reimão et al. 2011).

In a previous report, eight clinically used 1,4-dihydropyridines demonstrated in vitro anti-leishmanial activity with $\mathrm{IC}_{50}$ values ranging from 5.35-176.24 $\mu \mathrm{M}$ (Reimão et al. 2010). Amlodipine, lercanidipine, nicardipine and nimodipine were among the most active CCBs against $L$. (L.) chagasi amastigotes. Based on previous studies, CCBs were selected for drug combination assays, but none of the tested CCBs increased pentamidine, amphotericin B or glucantime in vitro activity.

Other promising pharmacological activities of CBBs have been reported in Leishmania sp., including the reversal of antimony resistance in clinical isolates of $L$. (L.) donovani (Valiathan et al. 2006). According to Misra et al. (1991), nifedipine and verapamil could effectively
A

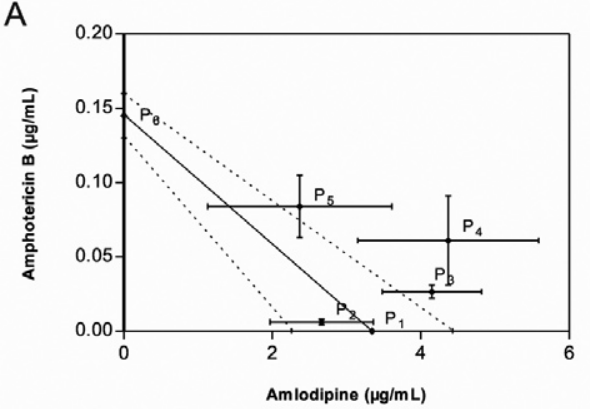

C

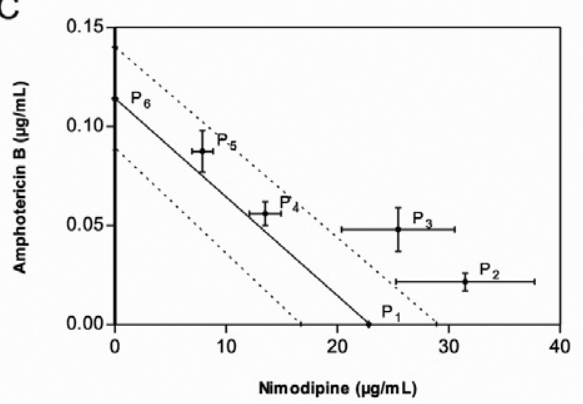

B

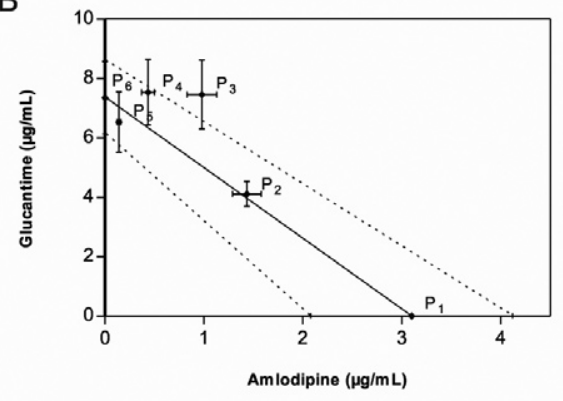

D

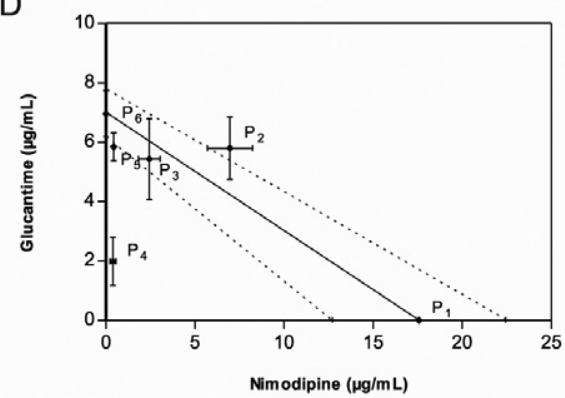

Fig. 2: representative isobolograms of in vitro interactions of calcium channel blockers (CCBs) and the partner drug against Leishmania (Leishmania) chagasi intracellular amastigotes. The half maximal inhibitory concentration $\left(\mathrm{IC}_{50}\right)$ of amlodipine and nimodipine was plotted in the abscissa and the $\mathrm{IC}_{50}$ of amphotericin $\mathrm{B}$ and glucantime was plotted in the ordinate. The plotted points are $\mathrm{IC}_{50} \mathrm{~s}$ of each fixed ratio combination serially diluted. Bars around points correspond to calculated standard errors of the mean. Points $\mathrm{P}_{1}-\mathrm{P}_{6}$ correspond to the proportion of 5:0, 4:1, 3:2, 2:3, 1:4 and 0:5 of CCB and standard anti-leishmanial drug, respectively. The bold line corresponds to the predicted positions of the experimental points for a simple additive effect. The dotted line corresponds to the additivity line range of confidence. 
inhibit the infection of macrophages by $L$. (L.) donovani, although no anti-parasitic effect could be observed. Palit and Ali (2008) also showed that lacidipine, amlodipine, verapamil and diltiazem inhibited $\mathrm{Ca}^{2+}$ uptake by Leishmania, but only lacidipine and amlodipine showed in vivo anti-leishmanial activity. Therefore, studying the action mechanisms of CCBs could prove useful for developing drug design studies and novel strategies for reversing the resistance of Leishmania spp.

In vitro combination assays are an advantageous and rational methodology for the screening of synergic drug combinations. However, they do not provide other important information, such as pharmacokinetic (Seifert et al. 2011) and pharmacodynamic interactions (Seifert \& Croft 2006). This can only be measured by animal assays. Considering the in vitro anti-leishmanial activity of the studied CCBs, further in vivo studies may be considered to evaluate possible drug interactions in VL.

\section{ACKNOWLEDGEMENTS}

To Dr Karin Seifert (London School of Hygiene and Tropical Medicine), for suggestions during paper preparation.

\section{REFERENCES}

Alvar J, Croft S, Olliaro P 2006. Chemotherapy in the treatment and control of leishmaniasis. Adv Parasitol 61: 223-274.

Basco LK, Le Bras J 1991. Plasmodium falciparum: in vitro drug interaction between chloroquine and enantiomers of amlodipine. Exp Parasitol 72: 262-270.

Berembaum MC 1978. A method for testing for synergism with any number of agents. $J$ Infect Dis 137: 122-113.

Bryceson A 2001. A policy for leishmaniasis with respect to the prevention and control of drug resistance. Trop Med Int Health 6: 928-934.

Chappuis F, Sundar S, Hailu A, Ghalib H, Rijal S, Peeling RW, Alvar J, Boelaert M 2007. Visceral leishmaniasis: what are the needs for diagnosis, treatment and control? Nat Rev Microbiol 5: 873-882.

Coura JR 2009. Present situation and new strategies for Chagas disease chemotherapy - a proposal. Mem Inst Oswaldo Cruz 104: 549-554.

Deloron P, Basco LK, Dubois B, Gaudin C, Clavier F, Le Bras J, Verdier F 1991. In vitro and in vivo potentiation of chloroquine against malaria parasites by an enantiomer of amlodipine. Antimicrob Agents Chemother 35: 1338-1342.

Fivelman QL, Adagu IS, Warhurst DC 2004. Modified fixed-ratio isobologram method for studying in vitro interactions between atovaquone and proguanil or dihydroartemisinin against drugresistant strains of Plasmodium falciparum. Antimicrob Agents Chemother 48: 4097-4102.

Gessner PK 1995. Isobolographic analysis of interactions: an update on applications and utility. Toxicology 105: 161-179.

Herwaldt BL 1999. Leishmaniasis. Lancet 354: 1191-1199.

Mahmoudi N, de Julián-Ortiz JV, Ciceron L, Gálvez J, Mazier D, Danis M, Derouin F, García-Domenech R 2006. Identification of new antimalarial drugs by linear discriminant analysis and topological virtual screening. J Antimicrob Chemother 57: 489-497.

Makioka A, Kumagai M, Ohtomo H, Kobayashi S, Takeuchi T 2001. Effect of calcium antagonists, calcium channel blockers and calmodulin inhibitors on the growth and encystation of Entamoeba histolytica and E. invadens. Parasitol Res 87: 833-837.

Misra S, Naskar K, Sarkar D, Ghosh DK 1991. Role of $\mathrm{Ca}^{2+}$ ion on Leishmania-macrophage attachment. Mol Cell Biochem 102: 13-18.
Motro M, Shemesh J, Grossman E 2001. Coronary benefits of calcium antagonist therapy for patients with hypertension. Curr Opin Cardiol 16: 349-355.

Núñez-Vergara LJ, Squella JA, Bollo-Dragnic S, Marín-Catalán R, Pino L, Díaz-Araya G, Letelier ME 1998. Isradipine and lacidipine: effects in vivo and in vitro on Trypanosoma cruzi epimastigotes. Gen Pharmacol 30: 85-87.

Odds FC 2003. Synergy, antagonism and what the chequerboard puts between them. J Antimicrob Chemother 52: 1.

Olliaro PL 2010. Drug combinations for visceral leishmaniasis. Curr Opin Infect Dis 23: 595-602.

Olliaro PL, Taylor RW 2003. Antimalarial compounds: from bench to bedside. J Exp Biol 206: 3753-3759.

Palit P, Ali N 2008. Oral therapy with amlodipine and lacidipine, 1,4dihydropyridine derivatives showing activity against experimental visceral Leishmaniasis. Antimicrob Agents Chemother 52: 374-377.

Reimão JQ, Colombo FA, Pereira-Chioccola VL, Tempone AG 2011. In vitro and experimental therapeutic studies of the calcium channel blocker bepridil: detection of viable Leishmania (L.) chagasi by real-time PCR. Exp Parasitol 128: 111-115.

Reimão JQ, Scotti MT, Tempone AG 2010. Anti-leishmanial and anti-trypanosomal activities of 1,4-dihydropyridines: in vitro evaluation and structure-activity relationship study. Bioorg Med Chem 18: 8044-8053.

Seifert K, Croft SL 2006. In vitro and in vivo interactions between miltefosine and other antileishmanial drugs. Antimicrob Agents Chemother 50: 73-79.

Seifert K, Munday J, Syeda T, Croft SL 2011. In vitro interactions between sitamaquine and amphotericin B, sodium stibogluconate, miltefosine, paromomycin and pentamidine against Leishmania donovani. J Antimicrob Chemother 66: 850-854.

Singh BN 1986. The mechanism of action of calcium antagonists relative to their clinical applications. Br J Clin Pharmac 21: 109S-21S.

Song HO, Ahn MH, Ryu JS, Min DY, Joo KH, Lee YH 2004. Influence of calcium ion on host cell invasion and intracellular replication by Toxoplasma gondii. Korean J Parasitol 42: 185-193.

Stauber LA, Franchino EM, Grun J 1958. An eight-day method for screening compounds against Leishmania donovani in golden hamster. J Protozool 5: 269-273.

Sundar S 2001. Drug resistance in Indian visceral leishmaniasis. Trop Med Int Health 6: 849-854.

Tada H, Shiho O, Kuroshima K, Koyama M, Tsukamoto M 1986. An improved colorimetric assay for interleukin 2. J Immunol Methods 93: 157-165.

Tallarida RJ, Kimmel HL, Holtzman SG 1997. Theory and statistics of detecting synergism between two active drugs: cocaine and buprenorphine. Psychopharmacology 133: 378-382.

Tanabe K, Izumo A, Kato M, Miki A, Doi S 1989. Stage-dependent inhibition of Plasmodium falciparum by potent $\mathrm{Ca}^{2+}$ and calmodulin modulators. J Protozool 36: 139-143.

Tempone AG, Pimenta DC, Lebrun I, Sartorelli P, Taniwaki NN, de Andrade HF Jr, Antoniazzi MM, Jared C 2008. Antileishmanial and antitrypanosomal activity of bufadienolides isolated from the toad Rhinella jimi parotoid macrogland secretion. Toxicon 52: 13-21.

Tempone AG, Taniwaki NN, Reimão JQ 2009. Antileishmanial activity and ultrastructural alterations of Leishmania (L.) chagasi treated with the calcium channel blocker nimodipine. Parasitol Res 105: 499-505.

Valiathan R, Dubey ML, Mahajan RC, Malla N 2006. Leishmania donovani: effect of verapamil on in vitro susceptibility of pro- 
mastigote and amastigote stages of Indian clinical isolates to sodium stibogluconate. Exp Parasitol 114: 103-108.

van Griensven J, Balasegaram M, Meheus F, Alvar J, Lynen L, Boelaert M 2010. Combination therapy for visceral leishmaniasis. Lancet Infect Dis 10: 184-194.
White NJ 1999. Delaying antimalarial drug resistance with combination chemotherapy. Parasitologia 41: 301-308.

Yardley V, Croft SL 2000. A comparison of the activities of three amphotericin B lipid formulations against experimental visceral and cutaneous leishmaniasis. Int J Antimicrob Agents 4: 243-248. 